\title{
The immediate influence of various whole-body vibration frequency on balance and walking ability in children with cerebral palsy: a pilot study
}

\author{
Yong-Gu Han, Soon-Won Lee, Chang-Kyo Yun* \\ Department of Physical Therapy, Daegu University Rehabilitation Medical Clinic, Daegu, Korea
}

The purpose of this pilot study is to examine the immediate effect of interventions based on the frequency of whole-body vibration on children with cerebral palsy's balance and walking abilities. A total of 12 were selected as subjects. All subjects measured 10-m walking test (10MWT), Timed Up and Go (TUG) test and measured sway length (SL) and limit of stability (LOS) by bio-rescue. And those were performed again after the frequencies of the 12,18 , and $26 \mathrm{~Hz}$ were applied respectively. To check the immediate effect, all subjects performed only one frequency of interventions per day and no other treatment was performed in parallel to control the foreign variables. After intervention, 10MWT were significantly increased in the 12 and $18 \mathrm{~Hz}$ groups $(P<0.05)$. TUG test and LOS were significantly increased in the $18 \mathrm{~Hz}$ group $(P<0.05)$. There were no significant differences on TUG, SL, and LOS in the $12 \mathrm{~Hz}$ group. There were no significant differences on 10MWT, TUG, SL, and LOS in the 26 $\mathrm{Hz}$ group. Looking at the results of this study, the immediate effect of whole-body vibration shows that the frequency of the $18 \mathrm{~Hz}$ group is the most effective interventions in promoting walking and balancing abilities in children with cerebral palsy than frequencies of the 12 and $26 \mathrm{~Hz}$ groups.

Keywords: Balance, Cerebral palsy, Walking speed, Whole-body vibration

\section{INTRODUCTION}

Children with cerebral palsy experience difficulty balancing and walking normally due to problems such as dysmyotonia and reduced selective control of voluntary muscles caused by brain damage (Campbell et al., 2013). Maintaining gait and balance is an integral part of engaging socially and functioning (Bonnechère et al., 2017; Cho et al., 2016); therefore, solving this problem is an important intervention goal for improving the quality of life and daily activities of children with cerebral palsy (Bonnechère et al., 2017; Moreau et al., 2016). Recently, the use of intervention devices designed for improving posture and gait control is increasingly prevalent among brain-injured patients (Khanuja et al., 2018).

Among these devices, the whole-body vibration (WBV) device is widely used to stimulate muscles throughout the body using the vibration stimulus of a foot plate and promote brain plasticity using the stimulation to somatosensory sensation in the sole (Kordi Yoosefinejad et al., 2015). The stretch reflex through WBV is effective for improving muscle endurance and balance control (Ritzmann et al., 2014). A recent meta-analysis of the literature suggested that children with cerebral palsy experienced greater improvement in physical function after 8- to 24-week long-term intervention followed by a combination of traditional physical therapy and WBV compared to children with cerebral palsy who only underwent traditional physical therapy (Saquetto et al., 2015).

However, despite various effects of WBV demonstrated in studies, the intervention has no set criteria for wave frequency, amplitude, or time in its application. In practice, wave frequency is an important factor in determining the intensity of exercise in the application of WBV (Lee et al., 2016) and it has been demonstrated that the muscle activation effect increases in proportion with
${ }^{*}$ Corresponding author: Chang-Kyo Yun (iD https://orcid.org/0000-0003-2094-4239 Department of Physical Therapy, Daegu University Rehabilitation Medical Clinic, 70 Myeongdeok-ro 8-gil, Nam-gu, Daegu 42400, Korea E-mail: puhaha1116@naver.com

Received: June 16, 2019 / Accepted: July 25, 2019
This is an Open Access article distributed under the terms of the Creative Commons Attribution Non-Commercial License (http://creativecommons.org/licenses/by-nc/4.0/) which permits unrestricted non-commercial use, distribution, and reproduction in any medium, provided the original work is properly cited. 
the WBV's wave frequency (Ritzmann et al., 2013). However, there is also a study in which training with an overly high wave frequency decreased balancing and walking speed in children with cerebral palsy due to muscle fatigue (Opheim et al., 2009).

Therefore, it is important to investigate the most effective wave frequency in the application of WBV for children with cerebral palsy to achieve the optimal walking speed and balance. When applying WBV, the 6-, 12-, 18-, and 26-Hz intensity levels are commonly used (Lee et al., 2016). However, there is a large variation in muscle activation according to the intensity level (Ritzmann et al., 2013) and in the research on the association between intensity level and physical ability in children with cerebral palsy. Therefore, this study's objective is to investigate the treatment effects of WBV wave frequency on walking speed and balance in children with cerebral palsy using various commonly used wave frequencies and thus determine the most effective intensity level.

\section{MATERIALS AND METHODS}

\section{Subjects}

This study was conducted as a pilot study. The subjects of this research are 12 children with cerebral palsy treated physical therapy and occupational therapy in Daegu university medical clinic. The program subjects were randomly selected from elementary, middle school, and high school students in Daegu. We selected 17 children prior to experiment. But, five children excluded. So, finally 12 children participated in this research. All subjects measured 10-m walking test (10MWT), Timed Up and Go (TUG) test and measured sway length (SL) and limit of stability (LOS) by bio-rescue from February 11, 2019 to February 23, 2019. After collecting the date on the baseline, the intervention of 12,18 , and $26 \mathrm{~Hz}$ was performed randomly in accordance with the order in which the decontamination was made from March 3, 2019 to March 15, giving a 10-min break to determine the immediate effect of the vibration training, and conducting the assessment again measure earlier. Only one of the 12-, 18-, and 26- $\mathrm{Hz}$ intervention were conducted per day, and no other treatment was used during the intervention period to exclude other foreign variables. All parents of subject have taken their written informed consent before the study. The selection criterion and exclusion criteria were as follows. Inclusion criteria: (a) children from 7 to 18 years old with diplegia or hemiplegia Cerebral palsy diagnostic, (b) cognitive development age higher than six, (c) Gross motor function classification scale (GMFCS) higher than three, (d) children who be able to maintain independent standing position. Exclu- sion criteria: (a) children who experienced leg fracture within a year, (b) children who had Botulinum toxin within 6 months, (c) children who eat medicine within 6 months.

\section{WBV program}

WBV training was performed on the Galileo vibration plate (Novotec, Pforzheim, Germany). The frequency was as follows. According to (Lee et al., 2016), the commonly used frequency are $6,12,18$, and $26 \mathrm{~Hz}$, but the frequency of 6 were excluded due to the lack of effect on the activation of muscles (Ritzmann et al., 2013), so frequency 12,18 , and $26 \mathrm{~Hz}$ were used as protocols in this study. In addition, each session consisted of three 3-min bouts of training, with a 3-min rest between WBV training (Gusso et al., 2015). To provide an only effect of $\mathrm{Hz}$, subject's posture was applied equally with a semisquitting position and an amplitude of $2.5 \mathrm{~mm}$ from the center of the platform (Stark et al., 2016).

\section{0-m walking test}

We analysis 10MWT for measuring the gait function. We marked the starting line and end line with tape on the floor, and children started slightly behind the line. Each subject completes the sequence 3 times, and the amount of time required for each sequence is measured and the average of the three is recorded. The test demonstrates high reliability with intraclass correlation of 0.81 for test-retest reliability in children with cerebral palsy (Thompson et al., 2008).

\section{Timed Up and Go test}

We analysis TUG test for measuring the dynamic balance. A child started in the sitting position at a chair. After stand and walked $3 \mathrm{~m}$, that children come back a chair and sit down. Each subject completes the sequence 3 times, and the amount of time required for each sequence is measured and the average of the three is recorded. Reliability of TUG test was high, with ICC of 0.99 for within-session reliability and 0.99 for test-retest reliability (Dhote et al., 2012).

\section{Bio-rescue}

Bio-rescue (RM Ingeierie, Rodez, France) was used for precise measurements using the machine along with clinical measures. Tasks are given using visual feedback from computer monitors connected to the decompression platform, and dynamic and static pressures through pressure sensors of the depressurization platform are measured. In order to familiarize themselves with the method of measurement, more than 4 years of skilled therapists 
performed the test first and then performed the measurement. The measurement method was performed in the standing position with the legs spread $30^{\circ}$ so that the second toe is on the radial line of the depressurization platform and the foreground. SL was performed to measure static balance, and LOS was performed to measure dynamic balance. Each subject completes the sequence 3 times, and the amount of time required for each sequence is measured and the average of the three is recorded. Bio-rescue showed good inter-rater and intra-rater reliability in measurements during footprint area (Kim et al., 2018).

\section{Statistical analysis}

G*Power package software program ver. 3.1.9.2 (Franz Faul., Universitat Kiel, Germany) was used to determine the required sample size for this study. All analyses were performing using IBM SPSS Statistics ver.21.0 (SPSS Inc., Chicago, IL, USA). Independent $t$-test was used to compare the general characteristics of subjects, such as age, height, and weight in subjects. One-way measure analysis of variance was used for analysis. The statistical significance level was set to $P<0.05$.

\section{RESULTS}

In this study, 12 children were tested. One out of every 17 existing children were excluded from the study because he was unable to participate in all experiments during the experiment period, while four children were excluded from this study outside the selection criteria. Three children were excluded in SL and LOS because they were unable to maintain their posture and perform tasks on the platform for a long time due to low attention. A diagram of the experimental procedure is shown in Fig. 1. The age, height, and weight of the subject did not show any significant difference within the group (Table 1). 10MWT in the 12 and 18

Table 1. The general characteristic of subjects

\begin{tabular}{lrccc}
\hline Characteristic & No. & Mean \pm SD & Range & $P$-value \\
\hline Age $(\mathrm{yr})$ & 12 & $12.08 \pm 3.26$ & $7.00-18.00$ & 0.82 \\
Height $(\mathrm{cm})$ & 12 & $143.25 \pm 15.93$ & $110.00-163.00$ & 0.60 \\
Weight $(\mathrm{kg})$ & 12 & $42.91 \pm 12.25$ & $25.00-60.00$ & 0.45
\end{tabular}

$\mathrm{SD}$, standard deviation.

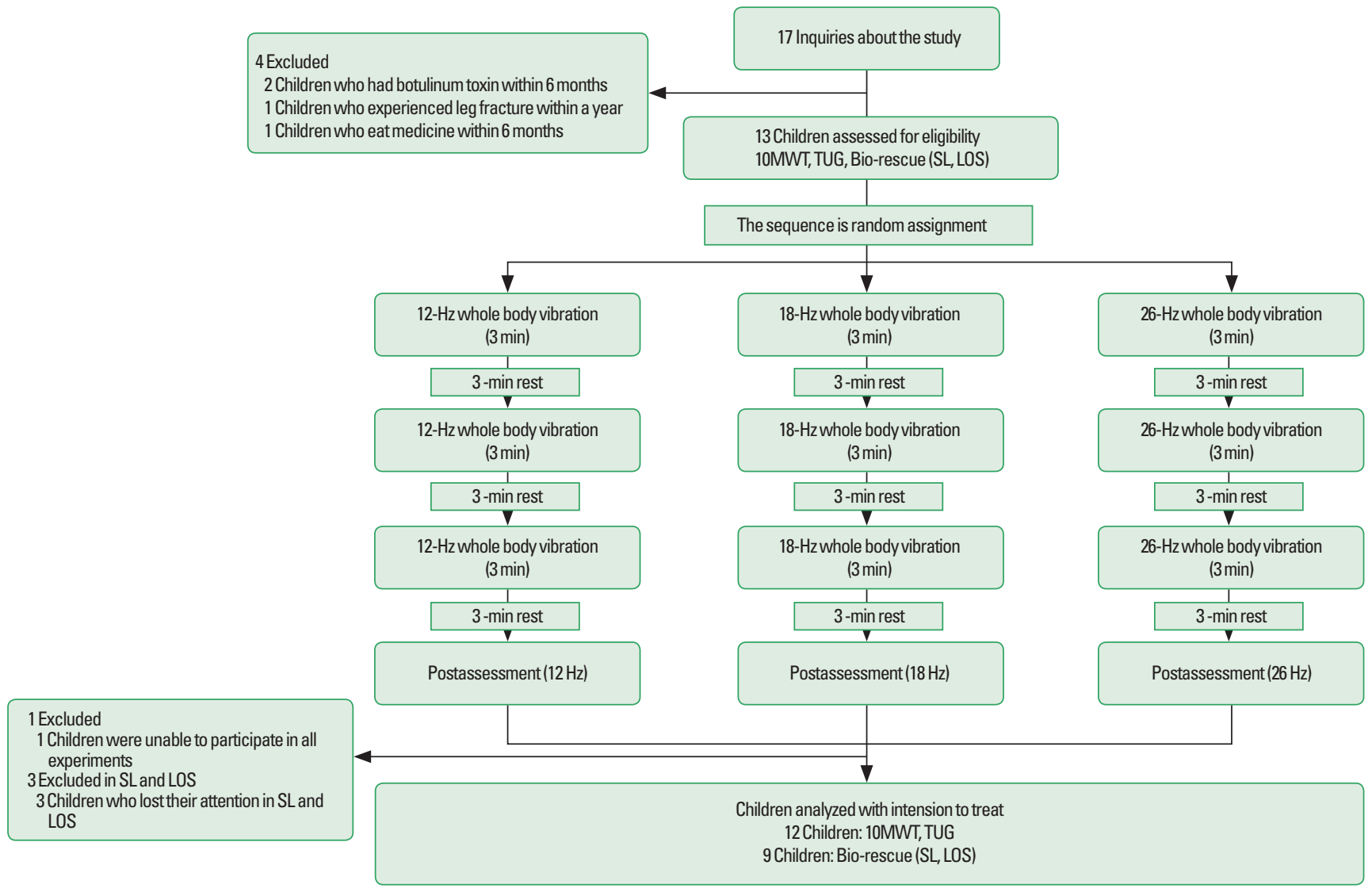

Fig. 1. Schematic diagram of study process. 10MWT, 10-m walking test; TUG, Timed Up and Go test; SL, sway length; LOS, limit of stability. 
Table 2. The change of many subjects following various vibration frequency

\begin{tabular}{|c|c|c|c|c|c|}
\hline Variable & Pre & $12 \mathrm{~Hz}^{\mathrm{a}}$ & $18 \mathrm{~Hz}^{\mathrm{bl}}$ & $26 \mathrm{~Hz}^{\mathrm{cl}}$ & $F$ \\
\hline 10MWT (cm/sec) & & & $(n=12)$ & & \\
\hline Mean \pm SD & $9.84 \pm 2.42$ & $9.30 \pm 2.40$ & $8.91 \pm 1.87$ & $9.36 \pm 2.36$ & 3.64 \\
\hline$P$-value & - & $0.02^{*}$ & $0.01^{*}$ & 0.16 & \\
\hline TUG $(\mathrm{cm} / \mathrm{sec})$ & & & $(n=12)$ & & \\
\hline Mean \pm SD & $10.99 \pm 3.86$ & $10.14 \pm 2.73$ & $9.24 \pm 1.83$ & $10.14 \pm 2.73$ & 3.34 \\
\hline$P$-value & & 0.11 & $0.04^{*}$ & 0.13 & \\
\hline $\mathrm{SL}(\mathrm{cm})$ & & & $(n=9)$ & & \\
\hline Mean \pm SD & $44.67 \pm 26.19$ & $59.93 \pm 57.33$ & $41.04 \pm 28.21$ & $44.50 \pm 17.38$ & 1.60 \\
\hline$P$-value & - & 0.21 & 0.26 & 0.98 & \\
\hline $\operatorname{LOS}\left(\mathrm{cm}^{2}\right)$ & & & $(n=9)$ & & \\
\hline Mean \pm SD & $3,344.56 \pm 2,700.03$ & $3,139.69 \pm 2,032.06$ & $4,059.00 \pm 2,642.64$ & $2,961.84 \pm 2,361.61$ & 5.25 \\
\hline$P$-value & - & 0.30 & $0.01^{*}$ & 0.80 & \\
\hline
\end{tabular}

Values are presented as mean \pm standard deviation.

SD, standard deviation; 10MWT, 10-m walking test; TUG, Timed Up and Go test; SL, sway length; LOS, limit of stability.

${ }^{*} \mathrm{P}<0.05$. ${ }^{\mathrm{a}} 12-\mathrm{Hz}$ vibration group, ${ }^{\mathrm{b}} 18-\mathrm{Hz}$ vibration group, ${ }^{\mathrm{c}} 26-\mathrm{Hz}$ vibration group.

$\mathrm{Hz}$ groups showed statistically significant difference after intervention $(P<0.05)$. TUG and LOS in the $18 \mathrm{~Hz}$ group showed statistically significant differences after intervention $(P<0.05)$ (Table 2). The $26 \mathrm{~Hz}$ group showed improved values although not a statistically significant difference. 10MWT in the $12 \mathrm{~Hz}$ group and TUG in the $26 \mathrm{~Hz}$ group showed improved values although not a statistically significant difference. SL in the $18 \mathrm{~Hz}$ group showed improved values although not a statistically significant difference. After intervention, there was no statistically significant difference on SL in the $12 \mathrm{~Hz}$ group instead numbers deteriorated. After intervention, there were no statistically significant differences on LOS in the 12 and $26 \mathrm{~Hz}$ groups instead numbers deteriorated (Table 2).

\section{DISCUSSION}

The results of this study demonstrate that the immediate effects of WBV are effective at improving balance and walking speed in children with cerebral palsy. In fact, improvements in leg strength and somatosensory stimulation are important therapeutic foci in efforts toward improved walking and balancing capabilities in children with cerebral palsy (Lee and Chon, 2013). Regarding research on the topic, Ritzmann et al. (2013) reported increased muscle activity and physiological responses after WBV intervention and that higher wave frequencies further increased muscle activity in electromyography and were more effective at improving leg strength. Moreover, vibration introduced to the subject's sole during WBV training induced the Hoffmann reflex and activated the spinal nerves to effectively stimulate the subject's inherent proprioceptive senses and somatosensory sensations (Apple et al., 2010). This likely explains the results of the present study, which suggest a statistically significant improvement in the 12 and 18 $\mathrm{Hz}$ group, and improved values in the $26 \mathrm{~Hz}$ group in the 10 MWT, a clinical gait scale that is performed with children with cerebral palsy after WBV intervention. The study results also showed a statistically significant improvement in the $18 \mathrm{~Hz}$ group and improved values in the other two groups on TUG, which is a clinical balance scale. In SL, $18 \mathrm{~Hz}$ group also showed improved values although not a statistically significant difference. The statistical and numerical improvements found in the present study are consistent with those reported in studies conducted with Parkinson patients (Ebersbach et al., 2008); the physiological mechanism of WBV mentioned earlier also likely contributed to walking speed and balance improvements in children with cerebral palsy.

However, the statistically significant and numerical improvements were greatest in the $18 \mathrm{~Hz}$ group for all measures. In particular, a statistically significant improvement on TUG and LOS was only found in the $18 \mathrm{~Hz}$ group and the numerical improvement on and SL was greatest in the $18 \mathrm{~Hz}$ group. This suggests that training at $18 \mathrm{~Hz}$ is a more effective intervention than at the higher intensity of $26 \mathrm{~Hz}$ for improving walking speed and balance in children with cerebral palsy at GMFCS stages 1 and 2. A potential reason for this result is the greater muscle fatigue among the $26 \mathrm{~Hz}$ group than the $18 \mathrm{~Hz}$ group due to the measurements being conducted immediately after a short intervention to determine their immediate effects. As demonstrated by a previous study that reported a decrease in jump height, oxygen saturation, 
and knee extension force due to muscle fatigue after a short WBV intervention at $26 \mathrm{~Hz}$ among college students (Rittweger et al., 2000), the fatigue that builds up in muscle fibers increases in proportion with exercise intensity (Stackhouse et al., 2005) and resistance to muscle fatigue is lower among children with cerebral palsy than the normal population (Moreau et al., 2008). Therefore, high-frequency WBV is presumed to have also affected immediate changes in function for children with cerebral palsy in the present study because it damages tissue and reduces muscle function before the muscle has fully recovered.

In contrast to the above results, there was no statistically significant difference at the measurement of LOS in 12 and $26 \mathrm{~Hz}$ groups. There was no statistically significant difference of SL in $12 \mathrm{~Hz}$ group; instead, numbers in the $12 \mathrm{~Hz}$ group deteriorated in SL and LOS and numbers in the $26 \mathrm{~Hz}$ group deteriorated in LOS. The potential reason for the result is as follows: Overly high wave frequency makes children of cerebral palsy feel significant fatigue and requires a long time for the muscle recovers, and is thought to have not recovered until an immediate assessment after intervention (Opheim et al., 2009). Also, low-intensity interventions are unlikely to induce muscle strength and functional improvement in children with cerebral palsy (Verschuren et al., 2016), and a short WBV intervention at low frequency has little effect on muscle activity in children with mild cerebral palsy at GMFCS stages 1 and 2 (Ritzmann et al., 2013). Moreover, low-intensity training for children reduces metabolic signals, induces cognitively adapted low-level efforts in assessment, and results in relatively slow recovery from muscle fatigue compared to high-intensity training (Ratel et al., 2006). Therefore, a short 12$\mathrm{Hz}$ intervention was likely insufficient stimulation to induce functional changes in children with cerebral palsy, yielding values that are somewhat confusing for the low-frequency intervention.

In conclusion, in WBV training with children with cerebral palsy, using the optimal frequency is important. In fact, for children with mild cerebral palsy at GMFCS stages 1 and 2, intervention with $18-\mathrm{Hz}$ frequency was more effective than that with $12-$ and $18-\mathrm{Hz}$ frequency in the study. As the study-maintained subjects' postures, vibration amplitudes, and intervention duration, extraneous variables were effectively controlled and the effect of maturity was also controlled because the immediate effects of WBV intervention were measured within a short-time period for the children with cerebral palsy. Therefore, as the measurements in the study only accounted for the effects of frequency, the independent variable, it can be concluded that the study has high validity and significance.
The study also has limitations. There were few subjects due to the clinical setting's characteristics. Therefore, the study results have limited generalizability to all patients with cerebral palsy. Moreover, the generalizability of the therapeutic intervention across functional levels also remains limited because subjects were primarily in GMFCS stage 1 or 2. Furthermore, it is also unknown how long the therapeutic effects last due to the lack of follow-up. Also, due to the lack of controls, the effects of this intervention compared to others or to no intervention are unknown. Finally, since this study is a pilot study, there is a limit to generalizing the results. Therefore, conducting further research by addressing the study limitations is recommended to help develop guidelines on the optimal WBV frequency for improved walking speed and balance in patients with cerebral palsy.

\section{CONFLICT OF INTEREST}

No potential conflict of interest relevant to this article was reported.

\section{REFERENCES}

Apple S, Ehlert K, Hysinger P, Nash C, Voight M, Sells P. The effect of whole body vibration on ankle range of motion and the H-reflex. $\mathrm{N}$ Am J Sports Phys Ther 2010;5:33-39.

Bonnechère B, Omelina L, Jansen B, Van Sint Jan S. Balance improvement after physical therapy training using specially developed serious games for cerebral palsy children: preliminary results. Disabil Rehabil 2017; 39:403-406.

Campbell L, Novak I, McIntyre S, Lord S. A KT intervention including the evidence alert system to improve clinician's evidence-based practice behavior--a cluster randomized controlled trial. Implement Sci 2013; 8:132.

Cho C, Hwang W, Hwang S, Chung Y. Treadmill training with virtual reality improves gait, balance, and muscle strength in children with cerebral palsy. Tohoku J Exp Med 2016;238:213-218.

Dhote SN, Khatri PA, Ganvir SS. Reliability of "modified timed up and go" test in children with cerebral palsy. J Pediatr Neurosci 2012;7:96-100.

Ebersbach G, Edler D, Kaufhold O, Wissel J. Whole body vibration versus conventional physiotherapy to improve balance and gait in Parkinson's disease. Arch Phys Med Rehabil 2008;89:399-403.

Gusso S, Colle P, Derraik JG, Biggs J, Munns C, Cutfield, W, Hofman P. Whole-body vibration training improves physical function and increases bone and muscle mass in youngsters with mild cerebral palsy. Horm Res Paediatr 2015;54:161-162. 
Khanuja K, Joki J, Bachmann G, Cuccurullo S. Gait and balance in the aging population: fall prevention using innovation and technology. Maturitas 2018;110:51-56.

Kim MK, Kim SG, Shin YJ, Choi EH, Choe YW. The relationship between anterior pelvic tilt and gait, balance in patient with chronic stroke. J Phys Ther Sci 2018;30:27-30.

Kordi Yoosefinejad A, Shadmehr A, Olyaei G, Talebian S, Bagheri H, Mohajeri-Tehrani MR. Short-term effects of the whole-body vibration on the balance and muscle strength of type 2 diabetic patients with peripheral neuropathy: a quasi-randomized-controlled trial study. J Diabetes Metab Disord 2015;14:45.

Lee BK, Chon SC. Effect of whole body vibration training on mobility in children with cerebral palsy: a randomized controlled experimenterblinded study. Clin Rehabil 2013;27:599-607.

Lee J, Lee K, Song C. Determining the posture and vibration frequency that maximize pelvic floor muscle activity during whole-body vibration. Med Sci Monit 2016;22:4030-4036.

Moreau NG, Bodkin AW, Bjornson K, Hobbs A, Soileau M, Lahasky K. Effectiveness of rehabilitation interventions to improve gait speed in children with cerebral palsy: systematic review and meta-analysis. Phys Ther 2016;96:1938-1954.

Moreau NG, Li L, Geaghan JP, Damiano DL. Fatigue resistance during a voluntary performance task is associated with lower levels of mobility in cerebral palsy. Arch Phys Med Rehabili 2008;89:2011-2016.

Opheim A, Jahnsen R, Olsson E, Stanghelle JK. Walking function, pain, and fatigue in adults with cerebral palsy: a 7-year follow-up study. Dev Med Child Neurol 2009;51:381-388.

Ratel S, Duché P, Williams CA. Muscle fatigue during high-intensity exercise in children. Sports Med 2006;36:1031-1065.
Rittweger J, Beller G, Felsenberg D. Acute physiological effects of exhaustive whole-body vibration exercise in man. Clin Physiol 2000;20:134142.

Ritzmann R, Gollhofer A, Kramer A. The influence of vibration type, frequency, body position and additional load on the neuromuscular activity during whole body vibration. Eur J Appl Physiol 2013;113:1-11.

Ritzmann R, Kramer A, Bernhardt S, Gollhofer A. Whole body vibration training--improving balance control and muscle endurance. PLoS One 2014;9:e89905.

Saquetto M, Carvalho V, Silva C, Conceição C, Gomes-Neto M. The effects of whole body vibration on mobility and balance in children with cerebral palsy: a systematic review with meta-analysis. J Musculoskelet Neuronal Interact 2015;15:137-144.

Stark C, Herkenrath P, Hollmann H, Waltz S, Becker I, Hoebing L, Semler O, Hoyer-Kuhn H, Duran I, Here B, Hadders-Algra M, Schoenau E. Early vibration assisted physiotherapy in toddlers with cerebral palsy-a randomized controlled pilot trial. J Musculoskelet Neuronal Interact 2016;16:183-192.

Stackhouse SK, Binder-Macleod SA, Lee SC. Voluntary muscle activation, contractile properties, and fatigability in children with and without cerebral palsy. Muscle Nerve 2005;31:594-601.

Thompson P, Beath T, Bell J, Jacobson G, Phair T, Salbach NM, Wright FV. Test-retest reliability of the 10-metre fast walk test and 6-minute walk test in ambulatory school-aged children with cerebral palsy. Dev Med Child Neurol 2008;50:370-376.

Verschuren O, Peterson MD, Balemans AC, Hurvitz EA. Exercise and physical activity recommendations for people with cerebral palsy. Dev Med Child Neurol 2016;58:798-808. 